University of Nebraska - Lincoln

DigitalCommons@University of Nebraska - Lincoln

Stephen Ducharme Publications

Research Papers in Physics and Astronomy

2012

\title{
The sweep rate dependence of the electrical control of magnetic coercivity
}

\author{
Abhijit Mardana \\ University of Nebraska-Lincoln, mardana.abhijit@gmail.com \\ Stephen Ducharme \\ University of Nebraska-Lincoln, sducharme1@unl.edu \\ Shireen Adenwalla \\ University of Nebraska at Lincoln, sadenwalla1@unl.edu
}

Follow this and additional works at: https://digitalcommons.unl.edu/physicsducharme

Part of the Physics Commons

Mardana, Abhijit; Ducharme, Stephen; and Adenwalla, Shireen, "The sweep rate dependence of the electrical control of magnetic coercivity" (2012). Stephen Ducharme Publications. 84.

https://digitalcommons.unl.edu/physicsducharme/84

This Article is brought to you for free and open access by the Research Papers in Physics and Astronomy at DigitalCommons@University of Nebraska - Lincoln. It has been accepted for inclusion in Stephen Ducharme Publications by an authorized administrator of DigitalCommons@University of Nebraska - Lincoln. 


\title{
The sweep rate dependence of the electrical control of magnetic coercivity
}

\author{
A. Mardana, Stephen Ducharme, and S. Adenwalla ${ }^{a}$ \\ Department of Physics and Astronomy and the Nebraska Center for Materials and Nanoscience, \\ University of Nebraska-Lincoln, Lincoln, Nebraska 68588-0299, USA
}

(Presented 2 November 2011; received 23 September 2011; accepted 31 October 2011; published online 28 February 2012)

\begin{abstract}
The sweep rate dependence of the coercive field in magnetic thin films is well-known. Here we present an experimental investigation of the dependence of electric field control of magnetization on the magnetic field sweep rate. Our sample is a ferroelectric/ferromagnetic heterostructure, with a soft polymer ferroelectric in contact with a transition metal ferromagnet, Co. Previous work on these heterostructures revealed a large change in coercive field and anisotropy when the ferroelectric polarization was switched from up to down. The present work shows that faster sweep rates increase the changes in coercive field that arise from switching the ferroelectric polarization. Our data indicate that the field sweep rate is an important parameter in the investigation of magnetoelectric coupling.

(C) 2012 American Institute of Physics. [doi:10.1063/1.3673829]
\end{abstract}

\section{INTRODUCTION}

In recent years, research on the electrical control of magnetic properties in composite materials via magnetoelectric coupling has been accelerating, leading to the discovery of exciting fundamental physics in addition to potential spintronics applications. Electric fields in magnetic thin films have been shown to control the magnetic anisotropy, ${ }^{1-5}$ the magnetization, the Curie temperature ${ }^{6-8}$ and the spin polarization. ${ }^{9}$ Ferroelectric/ferromagnetic heterostructures provide an easy route for the application of large electric field; however, in the majority of studies the strain mediated coupling ${ }^{10,11}$ between the components overwhelms the investigation of direct electric field induced effects. ${ }^{12,13}$ We have previously measured large polarization induced changes in the magnetic coercivity and anisotropy of thin Co films in a $\mathrm{Co} / \mathrm{P}(\mathrm{VDF}-\mathrm{TrFE})$ bilayer ${ }^{14} \mathrm{P}(\mathrm{VDF}-\mathrm{TrFE})$ is a ferroelectric copolymer with a large polarization and a stiffness coefficient that is approximately two orders of magnitude below that of Co. This combination produces a large electric field of $8.84 \times 10^{8} \mathrm{~V} / \mathrm{m}$ at the surface of the Co but very little, if any, strain in the Co layer, thereby enabling the investigation of purely electric field effects.

Our previous results ${ }^{14}$ can be summarized as follows. The polarization of the polymer ferroelectric has a significant effect on the coercivity and magnetic anisotropy of the thinnest Co films. The out-of-plane coercivity is significantly larger for up polarization (i.e. for the polarization pointing away from the Co layer), whereas the opposite is true for the in-plane coercivity. Magnetic anisotropy can be altered by as much as $50 \%$ by switching the ferroelectric polarization from up to down as calculated from in-plane and out-ofplane hysteresis loops. For the thinnest films, the easy magnetization axis switches from out-of-plane to in-plane as the ferroelectric polarization is switched. The change in coercivity is proportional to the ferroelectric polarization, as

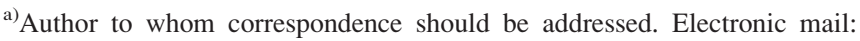
sadenwalla1@unl.edu.
}

confirmed by taking magnetization loops at intermediate polarization values. The magnetization can be rotated through a large angle using only electric fields; this rotation is electrically irreversible because the electric field changes the free energy of the thin ferromagnetic film. Experiments above the FE ordering temperature of the P(VDF-TrFE), at which no changes in the coercivity are seen, confirm that these large changes in the anisotropy arise from the large electric field at the surface of the Co layer, created by the presence of the polarized ferroelectric.

The ability to change the magnetic coercive field using an electric field has tremendous potential applications in magnetic recording media because it enables electric field writing. This present work was motivated by the well-known dependence of the magnetic coercivity on the magnetic field sweep rate, prompting us to investigate how the polarization induced changes in coercivity, $\Delta \mathrm{H}_{\mathrm{c}}$, behave as a function of magnetic field sweep rate. As we shall show below, the results even at fairly slow sweep rates and over a small range are quite striking.

The sweep rate dependence of magnetic coercivity is well known and is most apparent in magnetic thin films. ${ }^{15-19}$ The magnetic coercivity decreases with the field sweep rate, approaching the intrinsic coercivity for very slow sweep rates. Because magnetization reversal occurs by thermally activated processes, magnetization switching times depend on the energy barrier to be overcome, an energy barrier that depends (among other things) on the externally applied field. Hence the coercive field, defined as the field at which half the sample volume has switched, ${ }^{15}$ is highly dependent on sweep rate. Because the exact dependence of $\mathrm{H}_{\mathrm{c}}$ on sweep rate varies with extrinsic film properties (grain size, roughness), our ferromagnetic/ferroelectric heterostructured sample consists of a single wedge shaped Co layer, in order to minimize these effects. In addition, we measured the sweep rate dependence of identically grown bare Co thin films to check for coercive field effects due to the P(VDF-TrFE) overlayer. 
(a)

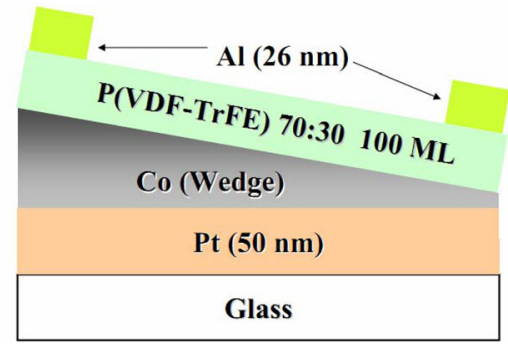

(b)

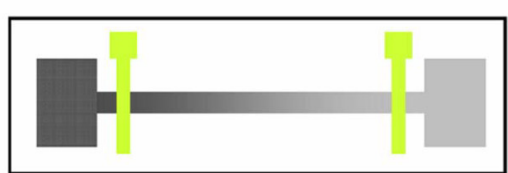

(c)

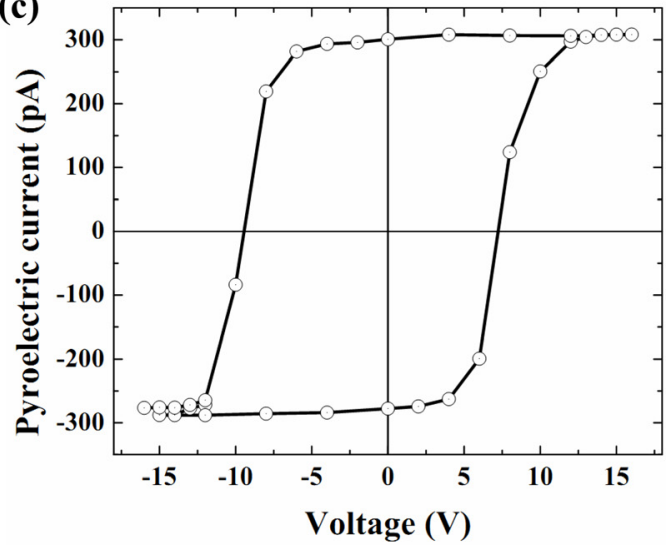

FIG. 1. (Color online) Sample schematic. (a) Cross sectional view of sample [B]: Glass/Pt $(50 \mathrm{~nm}) / \mathrm{Co}(2.5 \AA-$ $21.5 \AA) /[\mathrm{P}(\mathrm{VDF}-\mathrm{TrFE})$ 70:30] 100 $\mathrm{ML} / \mathrm{Al}(26 \mathrm{~nm})$. The Co wedge angle was $2.7 \times 10^{-6}$ degree. (b) Top view of the sample. LMOKE and PMOKE were done on the thick and thin edge respectively as shown in the diagram. (c) Ferroelectric polarization hysteresis loop as measured by the pyroelectric current versus applied voltage.

\section{EXPERIMENTAL PROCEDURES}

The samples consisted of [A] a set of bare Co films of varying thicknesses $(3 \mathrm{~nm}, 5 \mathrm{~nm}$ and $10 \mathrm{~nm})$ on a $50 \mathrm{~nm} \mathrm{Pt}$ seed layer and, [B], one sample with a ferroelectric overlayer consisting of Glass/Pt $(50 \mathrm{~nm}) / \mathrm{Co}(2.5 \AA-21.5 \AA) /[\mathrm{P}(\mathrm{VDF}-$ TrFE) 70:30] $100 \mathrm{ML} / \mathrm{Al}(26 \mathrm{~nm})$ as shown in Fig. 1. The wedge angle of the Co layer in this latter sample [B] is $2.7 \times 10^{-6}$ degree. All samples were grown on glass substrates. The Co and Pt layers were deposited by sputtering through shadow masks that were $1 \mathrm{~mm}$ wide and $15 \mathrm{~mm}$ long (for set [A)]) and $0.5 \mathrm{~mm} \times 40 \mathrm{~mm}$ long for the wedge shaped Co layer [B]. The deposition rates of $\mathrm{Co}$ and $\mathrm{Pt}$ were $0.2 \AA / s$ at $2 \times 10^{-3}$ Torr argon pressure.

The $180 \mathrm{~nm}$ thick ferroelectric polymer layer on the wedge-shaped Co was grown ex-situ by the LangmuirBlodgett (LB) technique and resulted in a crystalline ferroelectric film. The sample was annealed at $135^{\circ} \mathrm{C}$ for one hour. Upper electrodes of $\mathrm{Al}$ ( $26 \mathrm{~nm}$ thick) were deposited by thermal evaporation through shadow masks of width $0.2 \mathrm{~mm}$ at discrete regions along the Co wedge. Copper wires were attached to the top and bottom electrodes with silver paste to enable the measurement and switching of the ferroelectric polarization. The ferroelectric layer has been characterized using the Chynoweth method with a laser power of $1 \mathrm{~mW}$ and in reference to an optical chopper frequency of $2 \mathrm{kHz}$. The pyroelectric current is measured using a lock-in amplifier with $1 \mathrm{~s}$ time constant. The pyroelectric loop is shown in Fig. 1(c). The magneto-optical Kerr Effect (MOKE) was used to characterize the in-plane and out of plane magnetic behavior of all samples in longitudinal and polar configurations respectively.

\section{RESULTS AND DISCUSSIONS}

The dependence of the magnetic coercivity on magnetic field step size as a function of film thickness is shown in Fig. 2. Since the delay between each step is identical for all measurements $(300 \mathrm{~ms})$, the step size may be taken as a proxy for sweep rate. Both step size and sweep rate are indicated on the horizontal axis, with the sweep rate ranging from $0.85 \mathrm{mT} / \mathrm{s}$ to $20.25 \mathrm{mT} / \mathrm{s}$. Measurements were made on the three bare Pt/Co samples as well as on two ends of the

\section{Sweep rate $(\mathrm{mT} / \mathrm{s})$}

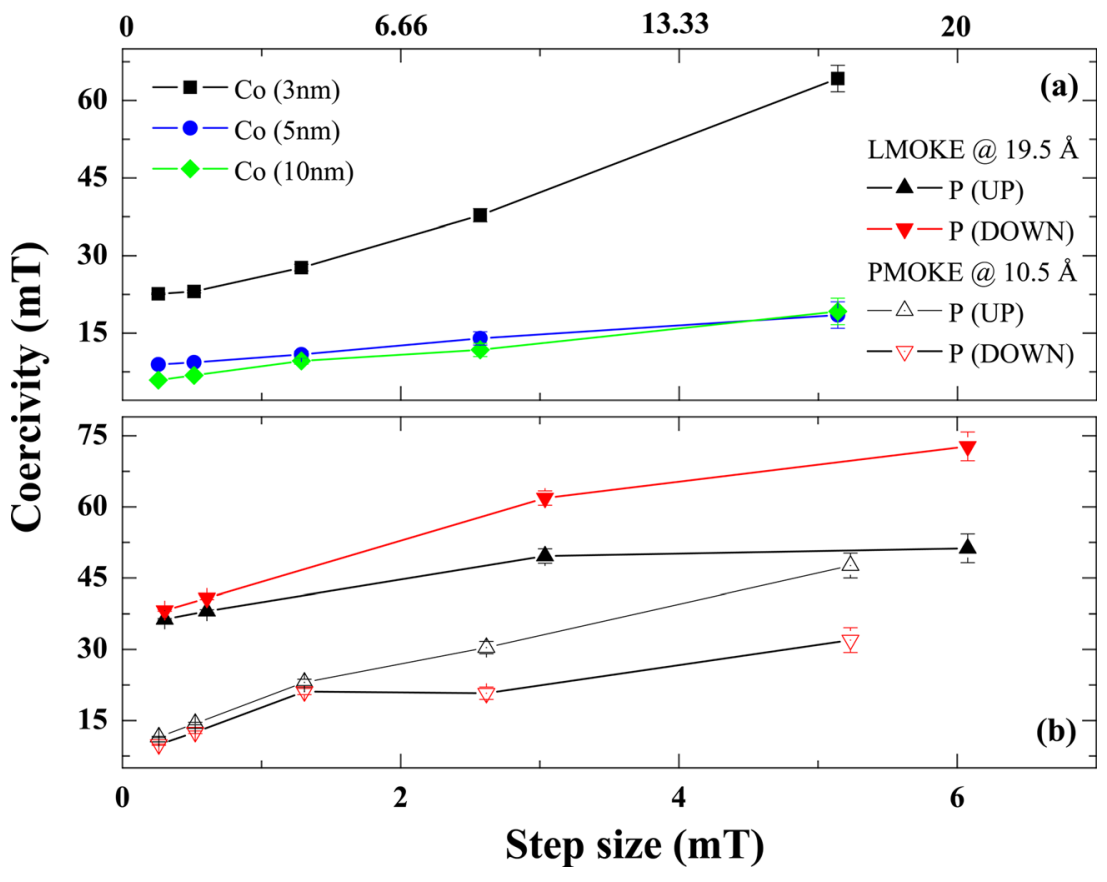

FIG. 2. (Color online) Magnetic coercivity as a function of sweep rate. (a) LMOKE measurements of sample set [A] consisting of bare Co on a Pt $(50 \mathrm{~nm})$ seed layer. With increasing Co thickness, the dependence of the coercivity on sweep rate decreases. (b) LMOKE and PMOKE measurements of sample [B]. The solid red and black data are for LMOKE measurements at a Co thickness of $19.5 \AA$ with the symbol direction depicting the FE polarization direction. The in-plane magnetic coercivity for ferroelectric polarization pointing down is always less than for polarization pointing up. The open red and black data are for PMOKE measurements at a Co thickness of $10.5 \AA$. The out-of-plane coercivity for up polarization is always less than for down polarization. The lines are guides to the eye. 


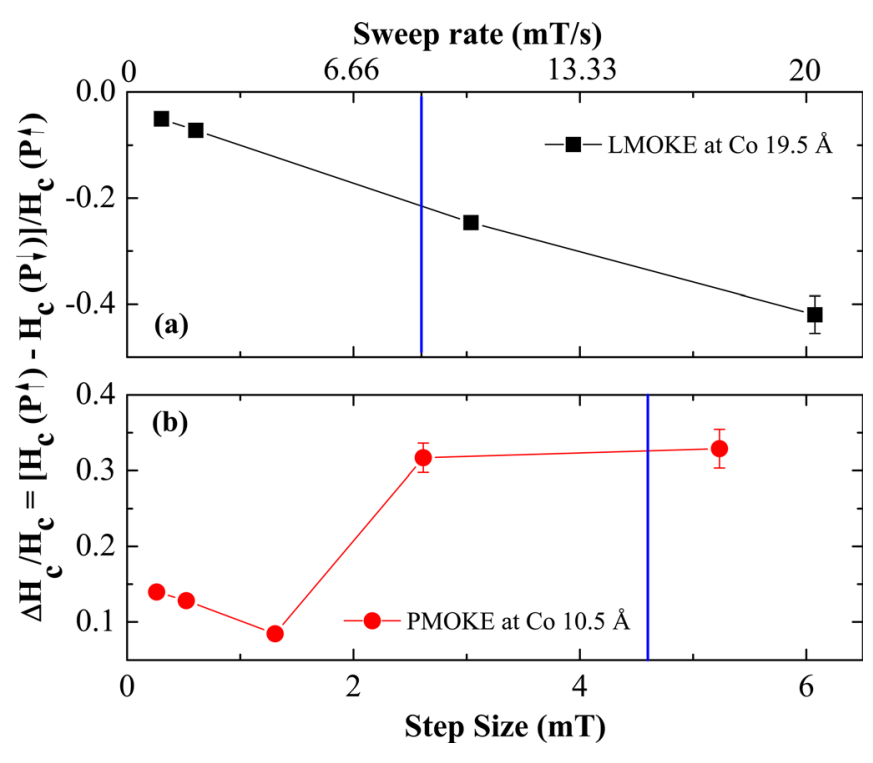

FIG. 3. (Color online) The normalized electric field induced difference in coercivity, $\Delta H_{c} / H_{c}$, as a function of magnetic field sweep rate, showing a substantial dependence. (a) In-plane LMOKE measurements for a Co thickness of $19.5 \AA$, and (b) out-of-plane PMOKE measurements for a Co thickness of $10.5 \AA$.

$\mathrm{Co} / \mathrm{P}(\mathrm{VDF}-\mathrm{TrFE})$ sample at positions along the Co wedge that correspond to thicknesses of $10.5 \AA$ and $19.5 \AA$. The out-of-plane (PMOKE) measurements were done at a Co thickness of $10.5 \AA$ and the in-plane (LMOKE) measurements were done at a Co thickness of $19.5 \AA$ corresponding to out-of plane and in-plane magnetization easy axes at the respective thicknesses. Over this rather restricted range of sweep rates, the thinnest sample of set [A], the $3 \mathrm{~nm}$ sample shows the maximum change in coercivity of $41.6 \mathrm{mT}$ whereas the $5 \mathrm{~nm}$ and $10 \mathrm{~nm}$ samples show changes of 16 $\mathrm{mT}$ and $14 \mathrm{mT}$ respectively, confirming the sweep rate dependence as well as the thickness dependence that has previously been observed. ${ }^{15-19}$ The coercivity of the ferromagnetic/ferroelectric sample is shown in Fig. 2(b), for both directions of polarization, with red and black data points indicating down and up polarization, respectively. The solid data points are for LMOKE measurements at a Co thickness of $19.5 \AA$ and the open data points are for PMOKE measurements for a Co thickness of $10.5 \AA$. The coercive field and its dependence on sweep rate are quite different from the expected dependence-with these thinner films, we expect a larger coercive field and much stronger sweep rate dependence than was obtained for the $3 \mathrm{~nm}$ bare Co film. We attribute this difference to chemical changes at the interface arising from the presence of the $\mathrm{P}(\mathrm{VDF}-\mathrm{TrFE})$. Our earlier work has shown a substantial shift of the spin reorientation transition when the $\mathrm{P}(\mathrm{VDF}-\mathrm{TrFE})$ layer is annealed and we expect that a similar phenomena is responsible for the coercive field data. As expected from our earlier measurements, the in-plane coercivity for up (pointing away from the Co surface) polarization is always smaller whereas for out-ofplane polarization, the reverse is true. Polarization switching was accomplished with an applied voltage of $\pm 20 \mathrm{~V}$ and all data are taken at zero applied electric field.
We are interested in how this difference in coercivity $\Delta H_{c}=H_{c}(\uparrow)-H_{c}(\downarrow)$, depends on the field sweep rate. This dependence is indicated in Fig. 3 in which the normalized difference $\Delta H_{c} / H_{c}(\uparrow)$ is plotted as a function of step size and sweep rate for both the in-plane data at a thickness of $19.5 \AA$ and the out-of-plane data at a thickness of $10.5 \AA$. The blue lines indicate the sweep rate of our previous experiments, albeit on different samples. There is a remarkably strong dependence of this change in coercivity on the sweep rate, all the more striking because the range of sweep rates used is quite modest. This dependence on the sweep rate implies that the electric field from the polymer must influence the magnetic domain structure, but the mechanism behind this remains unclear. Domain motion with the application of electric fields has been previously observed in $\mathrm{Fe}_{0.7} \mathrm{Ga}_{0.3} / \mathrm{BaTiO}_{3}{ }^{20}$ and $\mathrm{CoFe} / \mathrm{BaTiO}_{3}$ layered samples, ${ }^{21}$ but in those samples, strain coupling between the two materials is shown to be the driver behind the domain wall motion. This is unlikely to be the case in our samples.

In conclusion, we have investigated the effect of sweep rate on the electric field driven changes in magnetic coercivity in a polymer ferroelectric/ferromagnetic bilayer. Because of the large mismatch in stiffness coefficients, the magnetic changes with ferroelectric polarization that are observed are solely due to the presence of the electric field, and not to strain. In this magnetoelectric heterostructure, there is a substantial sweep rate dependence of the electric field driven changes in magnetic behavior, even over the small range of fairly slow sweep rates investigated. Because most studies of the electric of magnetism have been reported in very thin magnetic films and because the dynamic effect in these thin films can be very large, the sweep rate is an important parameter in any measure of magnetoelectric coupling in heterostructured thin films.

\section{ACKNOWLEDGMENTS}

This research is supported by the National Science Foundation through the MRSEC program under Grant No. DMR-0820521 and ECCS Grant No. 1101256.

${ }^{1}$ C. G. Duan et al., Appl. Phys. Lett. 92, 122905 (2008).

${ }^{2}$ K. Nakamura et al., Phys. Rev. B 80, 172402 (2009).

${ }^{3}$ J. M. Rondinelli et al., Nat. Nanotechnol. 3, 46 (2008).

${ }^{4}$ C. G. Duan et al., Phys. Rev. Lett. 101, 137201 (2008).

${ }^{5}$ T. Maruyama et al., Nat. Nanotechnol. 4, 158 (2009).

${ }^{6} \mathrm{H}$. Ohno et al., Nature 408, 944 (2000).

${ }^{7}$ T. Diet et al., Science 287, 1019 (2000).

${ }^{8}$ D. Chiba et al., Science 301, 943 (2003).

${ }^{9}$ V. Garcia et al., Science 327, 1106 (2010).

${ }^{10}$ S. Sahoo et al., Phys. Rev. B 76, 092108 (2007).

${ }^{11}$ N. A. Spaldin et al., MRS Bulletin 33, 1047 (2008).

${ }^{12}$ T. Cai et al., Phys. Rev. B 80, 140415(R) (2009).

${ }^{13}$ S. Zhang Phys. Rev. Lett. 83, 640 (1999).

${ }^{14}$ A. Mardana et al., Nano Lett. 11, 3862 (2011).

${ }^{15}$ M. P. Sharrock, J. Appl. Phys. 76, 10 (1994).

${ }^{16}$ P. Bruno et al., J. Appl. Phys. 68, 11 (1990).

${ }^{17}$ W. Kleemann, Annu. Rev. Mater. Res. 37, 415 (2007).

${ }^{18}$ M. P. Sharrock et al., IEEE Trans. Magn. 17, 3020 (1981).

${ }^{19}$ R. W. Chantrell et al., J. Phys. D: Appl. Phys. 21, 1469 (1988).

${ }^{20}$ T. Brintlinger et al., Nano Lett. 10, 1219 (2010).

${ }^{21}$ T. H. E. Lahtinen et al., Electric-field control of magnetic domain wall motion and local magnetization reversal, arXiv:1109.5514v1. 